\title{
INTERFERENCE WITH ASTRONOMICAL OBSERVATIONS OF OH MASERS FROM THE SOVIET UNION'S GLONASS SATELLITES
}

\author{
JOHN GALT \\ Dominion Radio Astrophysical Observatory, Penticton, BC, CANADA
}

Measurements at the $1612 \mathrm{MHz} \mathrm{OH}$ line are necessary to identify and study an important class of highly evolved stars whose properties cannot be determined by other means. Observing at this frequency over an 18 month period has been a frustrating experience in "sharing" a small portion of the $1610-1626.5 \mathrm{MHz}$ frequency allocation on a secondary basis with the Aeronautical Radionavigation Service. Transmissions from the GLONASS satellite system are so powerful that they are received in the side lobes and back lobes of the radio telescope, no matter where in the sky it is pointing as long as one satellite is above the horizon. On most days about half the observations had to be discarded to prevent spurious satellite signals from masquerading as astronomical spectral lines.

The radio interference was encountered during a systematic survey of infrared sources originally discovered by the IRAS satellite (Galt and Kwok, 1988 , van den Bergh, 1988). Many of these sources are cool stars surrounded by dust clouds in which narrow-band masers emit at a frequency near $1612.2 \mathrm{MHz}$. The maser emission is generated in a thin expanding shell centered on the star. A typical spectrum of such an object is shown in Figure 1 where the two peaks represent the approaching and receding surfaces of a shell, separated by a frequency corresponding to twice the radial velocity of expansion of the shell. Most of the sources are extremely weak, requiring many hours of integration for an unambiguous detection.

The astronomical importance of these measurements has been discussed elsewhere (see, for example, Herman and Habing, 1985). When combined with high resolution maps of the same shells using large array telescopes such as MERLIN or the VLA, distances to the stars can be accurately determined.

When interference is expected, it is necessary to split the observations into short, independent intervals in order that contaminated records can be rejected before final averaging. Figure 2 shows a sequence of thirty 11 -minute observations of which the first few are apparently free of interference. These spectra were produced by a frequency switching method in which the first local oscillator of the receiver was switched every 8 seconds to place the reception band first on the astronomical line and then on a reference region, usually one bandwidth lower in frequency. The "reference" spectra were then subtracted from the "line" spectra to produce the difference spectra shown. The last eighteen spectra of the sequence had to be discarded because of interference from GLONASS satellites. 
Figure 2 illustrates various ways in which the GLONASS signals contaminate astronomical spectra. It is obvious that records 74 to 90 inclusive must be rejected. For records 71 to 73 the situation is less clear. If many records such as 71 or 72 are accepted, there is a danger that weak features appearing in the final average may be spurious. Records 74 and 75 and perhaps 76 show a complicated frequency structure in the interference, perhaps caused by frequency-hopping modulation. Records 77 to 90 show large distortions in the spectra caused by the $(\sin x / x)^{* *} 2$ shape of the GLONASS signal. Records 87,88 and 89 show a strong $\mathrm{CW}$ signal which, if not rejected, could easily be mistaken for an astronomical OH-maser line.

Attempts have been made to "see through" the interference. One technique was to zero or clip low-order correlation coefficients before Fourier transformation. Another was to subtract a high-order polynomial from each spectrum. Neither method proved satisfactory. The signal-to-noise ratio on such "filtered" spectra was always worse than on similar spectra observed when no interference was present. This is not surprising considering spectra such as 74 and 75 . In some marginal cases such as 72,77 or 78 , the methods might be justified but, as emissions from the satellite do not have the characteristics of white noise, accepting such spectra could produce misleading astronomical results.

Another manifestation of the satellite interference can be seen in Figure 3 which shows a typical total power record made during a 16 hour observation of an $\mathrm{OH} / \mathrm{IR}$ star. The noise level in the absence of interference is less than the width of the pen line. Between 15:30 and 19:30 PST three separate satellites can be distinguished. The interference between 3:00 and 8:00, though at a lower level, is still strong enough to contaminate astronomical observations.

Spectra and other characteristics of the GLONASS signals were studied by Pankonin et al. in 1985 and later by Carter $(1986,1987)$. They estimated the strength of a single GLONASS satellite to be $40 \mathrm{~dB}$ above $(10,000$ times) the strength of Cassiopeia $A$, the strongest natural radio source in the sky. Other characteristics of the GLONASS system, determined at the University of Leeds, have been described by Klass (1987).

After the astronomical survey of $\mathrm{OH} / \mathrm{IR}$ sources was completed, a few days were devoted to looking directly at individual satellites in the GLONASS system to determine which of them were transmitting. Table I shows the results of this investigation. Of the 28 satellites for which we had orbital elements, eleven were transmitting for at least some of the time during astronomical observations. These identifications were made by measuring the central frequency of the interfering signal and comparing it with the frequencies determined by Daly (Dale and Daly 1986; Daly 1988a; Daly 1988b; Dale, Daly and Kitching 1988).

Spectra of the GLONASS signals were obtained in a few instances when pointing the antenna directly at the satellite. One such spectrum is shown in Figure 4. This spectrum has the same general form as those obtained by Pankonin et al. (1985) and by Carter $(1986,1987)$. The shaded area of the graph indicates the $1610.6-1613.8 \mathrm{MHz}$ region of the spectrum allocated to the radio astronomy service on a secondary basis for spectral line observations by the International Telecommunication Union's Radio Regulations. This allocation includes footnote 734 which states "...In making assignments to stations of other services to which the band is allocated, administrations are urged to take all 
practicable steps to protect the radio astronomy service from harmful interference..." It is obvious that this footnote was ignored by the authorities who assigned frequencies for the GLONASS satellites. Practicable steps which could have been taken include filtering of sidebands and avoidance of center frequencies in and near the allocated radio astronomy band. We assume that the designers of the GLONASS system were unaware of the need to protect the radio astronomy band and therefore did not appreciate the need for filtering. As the present system is just experimental, it would still be possible to protect the radio astronomy band when or if operational satellites are planned.

The transmitting satellites are, at present, spaced in orbits in such a way that several hours a day are interference-free or almost so. Thus astronomical measurements can still be made but at very low efficiency. According to papers presented to the International Civil Aviation Organization (Anodina, 1988) it is the Soviet Union's intention to have the complete system of 24 operational satellites (including 3 spares) in orbit by 1995. If this plan is followed without adequate protection for the radio astronomy service, no further astronomical measurements will be possible at this important frequency.

What can be done? The 20th General Assembly of the International Astronomical Union has passed a resolution which will bring the matter to the attention of the International Telecommunication Union (IAU, 1988). It is hoped, but it is by no means certain, that the next World Administrative Radio Conference of the ITU will change the frequency allocations to clear all transmissions from the vicinity of the $1612 \mathrm{MHz} O H$ line as has already been done for the hydrogen line at $1420 \mathrm{MHz}$. Presumably, this would be possible only if the USA and the USSR agreed to deploy a single satellite navigation system which would avoid the astronomically important $1612 \mathrm{MHz}$ line. There would be obvious economic and operational advantages to a single, universally accepted, navigation system.

In the new spirit of glasnost, and with the end of the "cold war" in sight, can we now expect a level of international understanding which will ensure the preservation of this small but unique spectral region?

\section{ACKNOWLEDGEMENTS}

I wish to thank W. Sizemore at the National Radio Astronomy Observatory, Green Bank, for providing a computer program to calculate satellite positions. The orbital elements of GLONASS satellites were generously provided by the Project Operations Branch of Goddard Space Flight Center.

I am indebted to P. Daly of the University of Leeds for information on frequencies of the various satellites. K. McKay assisted with the spectral observations of the satellites. O. B. Aaquist experimented with clipping and zeroing low order correlation coefficients in an attempt to reduce the effects of broad band interference.

\section{REFERENCES}

Anodina, T. G., 1988, "The Glonass System Technical Characteristics and Performance," Special Committee on Future Air Navigation of the 
International Civil Aviation Organization, FANS/4-WP/75 (see also WP-38 and WP-70).

Carter, J. C., 1986, "Glosnass Observations" (Haystack memorandum), also issued as VLA Test Memorandum No. 150, National Radio Astronomy Observatory, Socorro, NM.

Carter, J. C., 1987, "Glonass Observations" (Haystack memorandum), also issued as VLA Test Memorandum No. 151, National Radio Astronomy Observatory, Socorro, NM.

Dale, S. A. and Daly, P., 1986, Recent Observations with the Soviet Union's Glonass Navigation Satellites, Position Location and Navigation Symposium, Las Vegas (IEEE PLANS '86) p. 20.

Dale, S. A., Daly, P. and Kitching, I. D., 1988, "Understanding Signals from Glonass Satellites," International Journal of Satellite Communications, Vol. 6, No. 3 (to appear).

Daly, P., 1988a, private communication.

Daly, P., 1988b, "Aspects of the Soviet Union's Glonass Satellite Navigation System," Journal of Navigation, Vol. 41, p. 186.

Galt, J. A. and Kwok, S., 1988, "A Search for $1.612 \mathrm{GHz}$ OH Emission from IRAS Sources," J. Roy Astron. Soc. Can. (in press).

Herman, J. and Habing, H. J., 1985, "Time Variations and Shell Sizes of $\mathrm{OH}$ Masers in Late-Type Stars," Astron. Astrophys. Suppl. Ser. 59, p. 523.

IAU, 1988, "Proceedings of the Twentieth General Assembly," of the International Astronomical Union, Baltimore, 1988, D. Reidel Publishing Company (in press).

Klass, P. J., 1987, "British Scientists Reveal Signal Format of Soviet Navsat," Aviation Week \& Space Technology, Apr. 13, p. 109.

Pankonin, V., Brundage, W. D., Carter, J. C. and Davis, M. M., 1985, "Interference to Radio Astronomy from Glonass Transmissions in the Frequency Band 1600 - $1615 \mathrm{MHz}$," VLA Test Memorandum No. 146, National Radio Astronomy Observatory, Socorro, NM.

van den Bergh, S., 1988, "The Effects of Space Debris and Satellite Interference on Astronomy," Proc. International Colloquium on "Environmental Aspects of Activities in Outer Space - State of the Law and Measure of Protection," Cologne, May 17. 
TABLE I Survey of GLONASS satellites 1988 May 10 to 17

\section{Satellites \\ observed to be transmitting:}

COSMOS

$\begin{array}{ll}1490 & 1413 \\ 1593 & 1414 \\ 1650 & 1491^{*} \\ 1651 & 1492 \\ 1710 & 1519^{*} \\ 1778 & 1520^{*} \\ 1779 & 1521 \\ 1780 & 1554^{*} \\ 1883 & 1555^{*} \\ 1884 & 1594 \\ 1885 & 1595^{*} \\ & 1652 \\ & 1711^{*} \\ & 1712 \\ & 1838 \\ & 1839 \\ & 1840\end{array}$

Satellites

which were NOT

transmitting:

COSMOS

413

$1491^{*}$

$1519^{*}$

$1520^{*}$

$1555^{*}$

1594

1652

$1711^{*}$

1712

1839

*These satellites were identified by the frequency of their transmissions during astronomical observations, not by their position in orbit. 


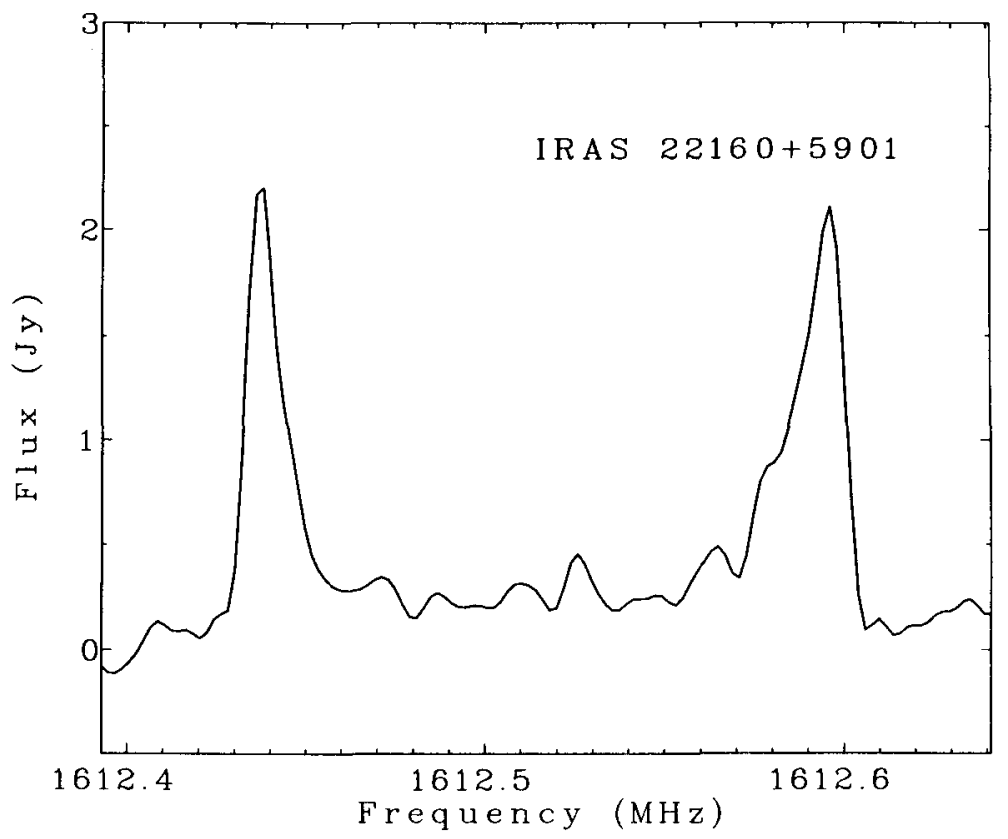

Figure 1. Spectrum of radio emission from the infrared source IRAS $22160+5901$ obtained during intervals when little or no GLONASS interference was present. 


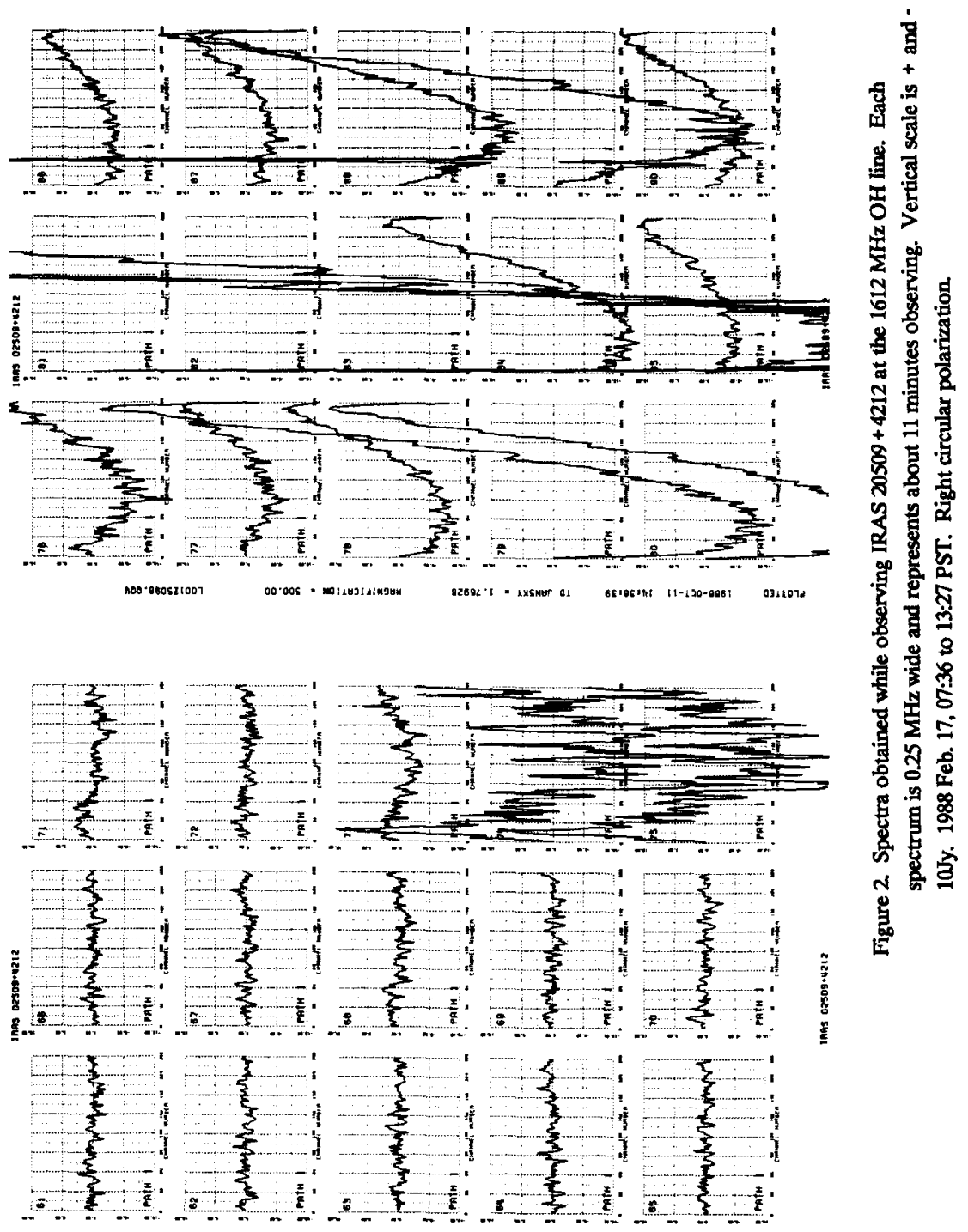




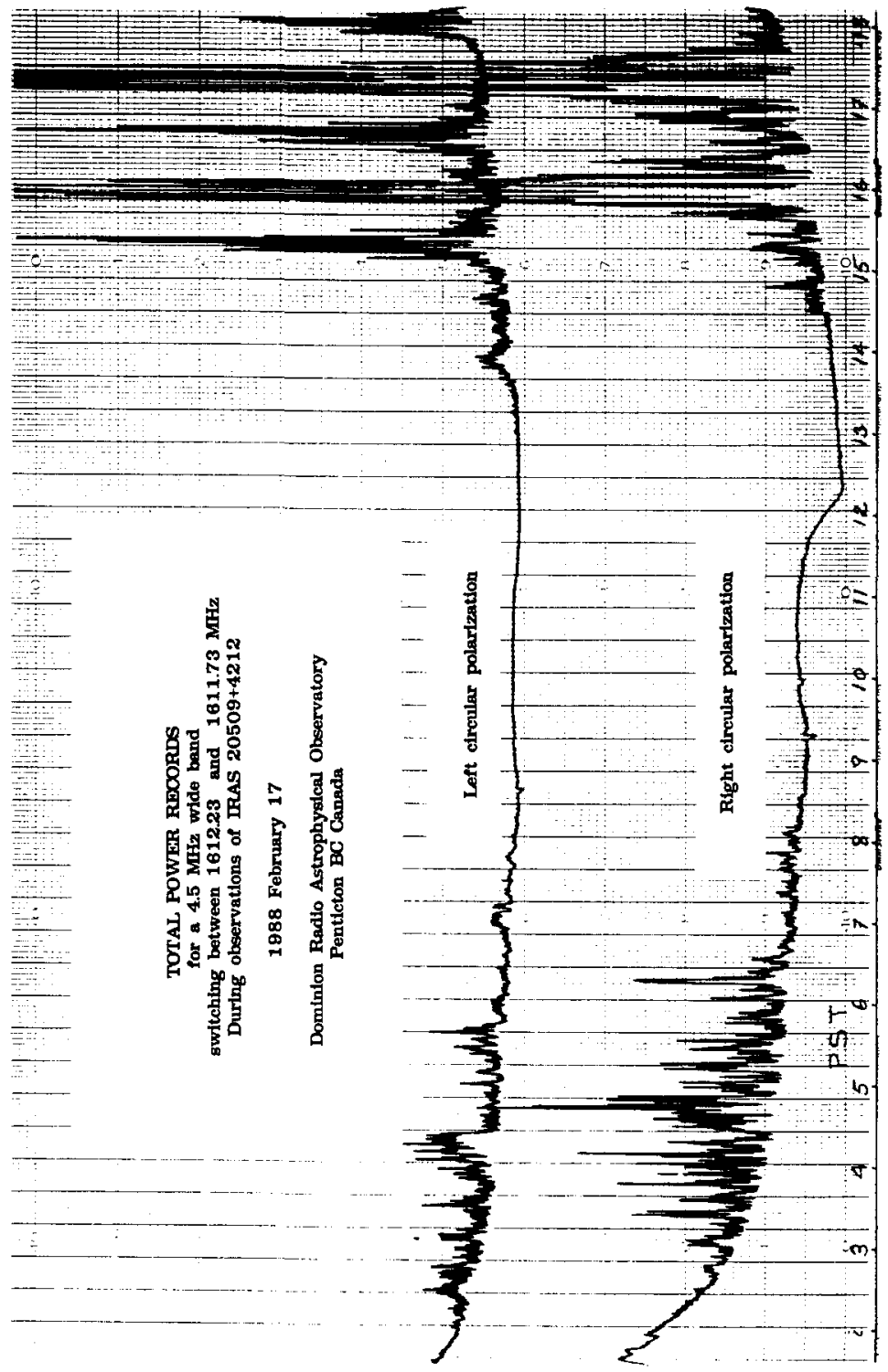

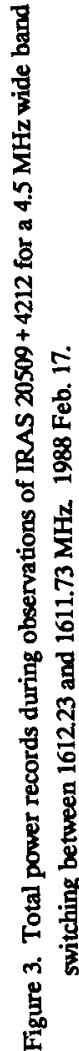




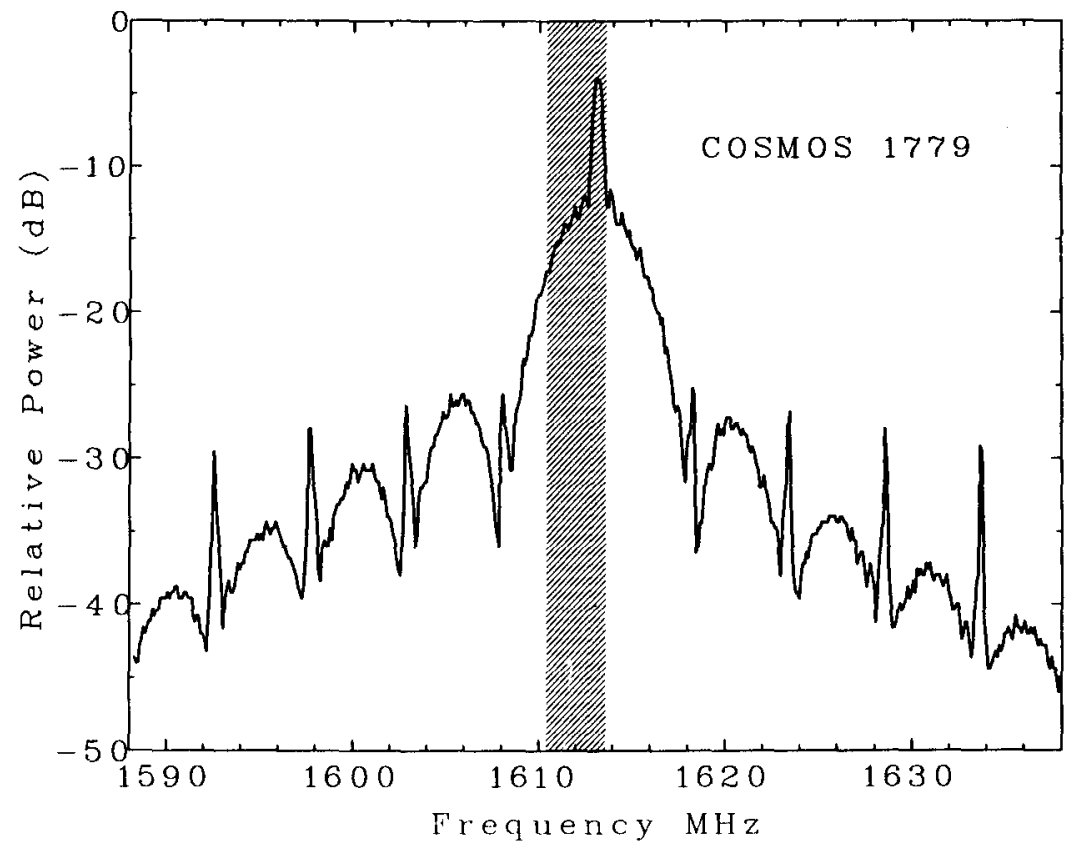

Figure 4. Spectrum of COSMOS 1779 obtained by pointing the antenna directly at the satellite. The shaded area represents the band allocated to the radio astronomy service by the International Telecommunication Union for spectral line observations of the hydroxyl radical (OH). 